\title{
Extração de cromo via processamento químico de finos de ligas Fe-Cr com alto teor de carbono
}

\author{
TORRES D.M. ${ }^{1}$, NAVARRO, R. C. S. ${ }^{1}$, SOUZA R. F. M. ${ }^{1}$, BROCCHI E. A. ${ }^{1}$ \\ ${ }^{1}$ PUC-Rio, Departamento de Engenharia de Materiais \\ E-mail para contato: douglas.metalurgia@gmail.com
}

\begin{abstract}
RESUMO: Ligas ferrosas são importantes insumos nos processos de obtenção de aços especiais. Neste contexto, ligas do sistema $\mathrm{Fe}-\mathrm{Cr}$, com teor de $\mathrm{Cr}$ típico variando entre 45 a 95\% em massa, apresentam destaque, principalmente na fabricação de aços inoxidáveis. Durante processo de obtenção das referidas ligas, há considerável produção de finos, os quais podem ser reaproveitados mediante tratamento químico (piro e hidrometalúrgico) apropriado. No presente trabalho investigou-se a extração de cromo de finos gerados durante a produção de uma liga $\mathrm{Fe}-\mathrm{Cr}$ com alto teor de $\mathrm{C}$. Para tanto foram realizadas ustulações nas temperaturas de 800,900 e $1000^{\circ} \mathrm{C}$ com $300 \%$ de excesso de $\mathrm{NaOH}$ em atmosfera oxidante, seguida de solubilização em meio aquoso. As recuperações de $\mathrm{Cr}$ em solução máximas alcançadas foram da ordem de $86 \%$, sugerindo que a rota de processamento químico proposta foi satisfatória no que tange à extração do cromo inicialmente presente.
\end{abstract}

\section{INTRODUÇÃO}

Ferro-ligas são importantes insumos para a produção de aços especiais. Dentre estas, ligas $\mathrm{Fe}-\mathrm{Cr}$ possuem um papel de destaque no que tange à fabricação de aços inoxidáveis, cujos teores típicos de cromo variam entre 11 e $25 \%$. Tal fato explica a significativa produção deste insumo, que de acordo com Sumario mineral brasileiro de (2012) atingiu o montante de 145,122 toneladas em 2011, distribuído entre ligas com alto teor de C (FeCrAC), com teor de $\mathrm{C}$ variando entre 4 e $10 \%$, e baixo teor de carbono (FeCrBC), com teores de até $0.5 \%$ (Vanhanem, 1999; Habashi, 1997). A obtenção de ligas Fe-Cr se dá principalmente por meio de dois processos, empregando-se como agentes redutores $\mathrm{C}$ ou $\mathrm{Si}$, sendo o primeiro associado à síntese de ligas FeCrAC, e o segundo às ligas FeCrBC (Wenzel, 2008; Habashi, 1997). No processo de produção de ligas FeCrAC são gerados dois tipos de materiais, o granulado, que possui tamanho de partícula já adequado para ser utilizado no refino secundário, e "finos", que, por apresentarem granulometria demasiadamente diminuta, não podem ser incorporados á carga da panela. No entanto, devido aos teores apreciáveis de $\mathrm{Cr}$ encontrados neste tipo de material, metal este de expressivo valor tecnológico, tem-se a motivação para o desenvolvimento de processos que viabilizem a recuperação do mesmo.

O presente trabalho tem como principal objetivo promover um estudo da recuperação do cromo presente em finos gerados durante a produção de uma liga $\mathrm{FeCrAC}$, mediante a combinação de processos piro e hidrometalúrgicos, que se inicia mediante uma fusão alcalina, de maneira a gerar seletivamente um sal portador de cromo, que é em seguida dissolvido em meio aquoso, permanecendo o ferro na forma de compostos insolúveis nas mesmas condições (ex. óxidos) (Wenzel, 2008; Habashi , 2002). O processo empregado no presente estudo é em 
essência constituído por duas etapas (tópico 3), fusão alcalina e concomitante ustulação (800 $1000^{\circ} \mathrm{C}$ ) em atmosfera oxidante na presença de $\mathrm{NaOH}$, para obtenção do cromato de sódio $\left(\mathrm{Na}_{2} \mathrm{CrO}_{4}\right)$, seguida de solubilização em água destilada a quente $\left(90-100^{\circ} \mathrm{C}\right)$, visando a extração seletiva do cromo inicialmente presente.

\section{FUNDAMENTAÇÃO TEÓRICA}

Mediante o emprego do software HSC 6.0, realizou-se um estudo da viabilidade termodinâmica da etapa de ustulação oxidante (Eq. 1), mediante a construção de curvas de variação de energia livre de Gibbs padrão como função da temperatura (Figura 1) e diagramas de distribuição de espécies (Figura $2 \mathrm{a}$ e $2 \mathrm{~b}$ ). Nesta apreciação, a presença da liga $\mathrm{Fe}-\mathrm{Cr}$ foi considerada através da inclusão de $\mathrm{Fe}$ e $\mathrm{Cr}$ na forma isolada, e de $\mathrm{C}$ na forma de $\mathrm{Cr}_{7} \mathrm{C}_{3}$. Adicionalmente, considerou-se a possível presença de $\mathrm{Fe}$ na forma de inclusões oxidadas $\left(\mathrm{Fe}_{2} \mathrm{O}_{3}\right)$ Malaga (2009). De acordo com os dados apresentados na Figura 1, observa-se que a reação de formação do cromato de sódio (Equação 1) apresenta significativa força motriz para temperaturas entre 200 e $1000^{\circ} \mathrm{C}$. A inclinação positiva da curva sugere uma expressiva redução da entropia do sistema durante o processo, a qual é motivada pelo consumo de 13.5 moles de $\mathrm{O}_{2}$, produzindo-se somente 5 moles de espécies gasosas $\left(\mathrm{CO}_{2}\right.$ e $\left.\mathrm{H}_{2} \mathrm{O}\right)$.

$$
4 \mathrm{Fe}+\mathrm{Cr}+\mathrm{Cr}_{7} \mathrm{C}_{3}+4 \mathrm{NaOH}+13.5 \mathrm{O}_{2(g)}+\mathrm{Fe}_{2} \mathrm{O}_{3}=2 \mathrm{Na}_{2} \mathrm{CrO}_{4}+3 \mathrm{Fe}_{2} \mathrm{O}_{3}+3 \mathrm{CO}_{2(g)}+2 \mathrm{H}_{2} \mathrm{O}_{(g)}+3 \mathrm{Cr}
$$

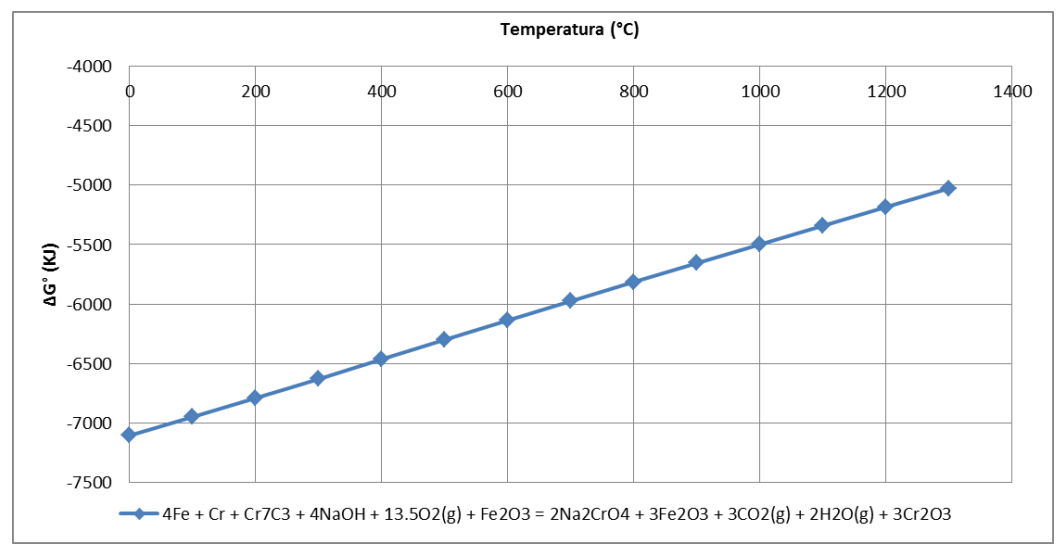

Figura 1 - Variação da energia livre de Gibbs para a reação de ustulação com NaOH.

Embora os valores expressivamente negativos associados à energia de Gibbs padrão da reação de ustulação (Figura 1) deem suporte à viabilidade termodinâmica do processo, este tipo de apreciação não contempla o estado de equilíbrio final alcançado, que pode ser investigado mediante a construção de diagramas de distribuição de espécies. Nesta abordagem, permite-se variar a relação molar inicial entre os reagentes envolvidos, calculando-se a concentração de todas as espécies acessíveis ao sistema reacional em dada temperatura. De acordo com os dados contidos na Figura 2a, observa-se que o óxido de cromo é a espécie predominante em toda faixa de temperatura investigada.

Desta forma, na tentativa de deslocar o equilíbrio no sentido da formação do $\mathrm{Na}_{2} \mathrm{CrO}_{4}$ foram adicionados excessos de $\mathrm{O}_{2}(\mathrm{~g})$ e $\mathrm{NaOH}$ em relação à condição estequiométrica, respectivamente iguais a $150 \%$ e $300 \%$. Ambas as condições favorecem a formação exclusiva da espécie $\mathrm{Na}_{2} \mathrm{CrO}_{4}$ (Figura 2b). 

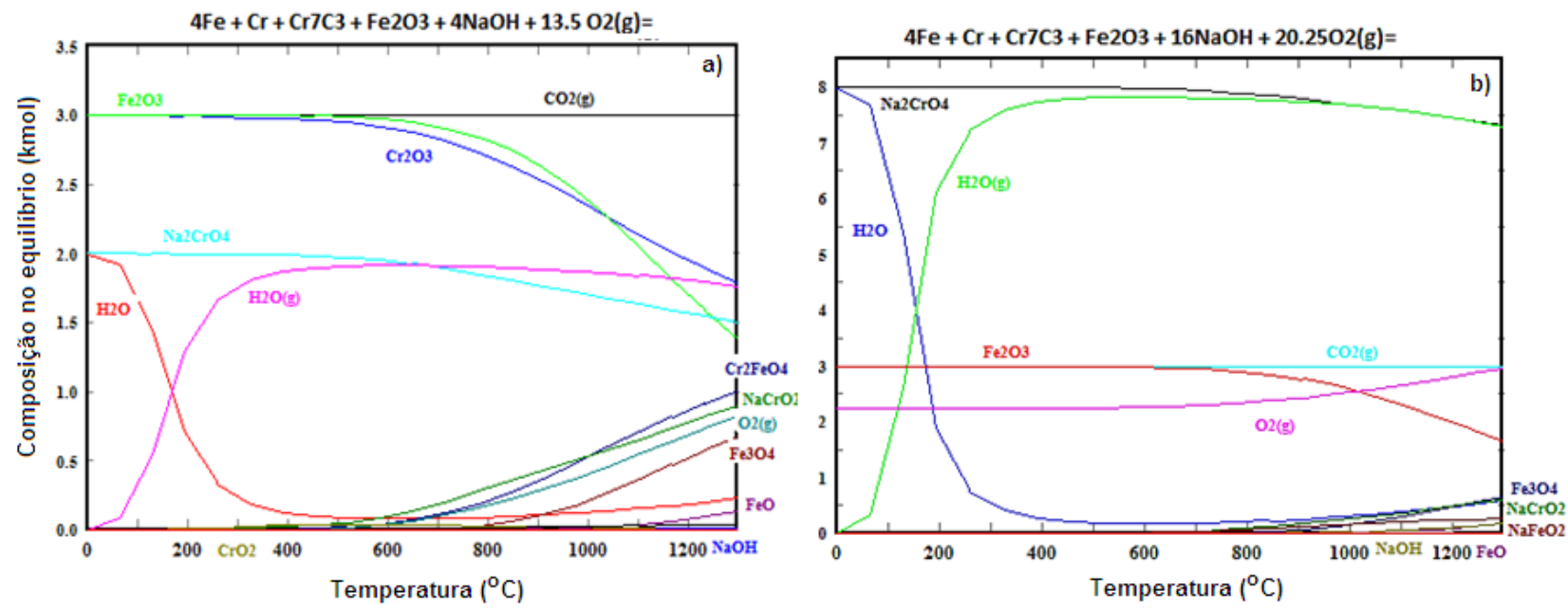

Figura 2 - (a) Composição estequiométrica, (b) Excesso de $\mathrm{NaOH}$.

Obtido o cromato de sódio é necessário solubilizá-lo em água destilada a quente (em torno de $100^{\circ} \mathrm{C}$ ). Para isso é de suma importância entender o comportamento das possíveis espécies iônicas portadoras de cromo em solução aquosa, que, por sua vez, pode ser estudado em uma primeira aproximação através da construção de diagramas de Pourbaix (Figura 3).

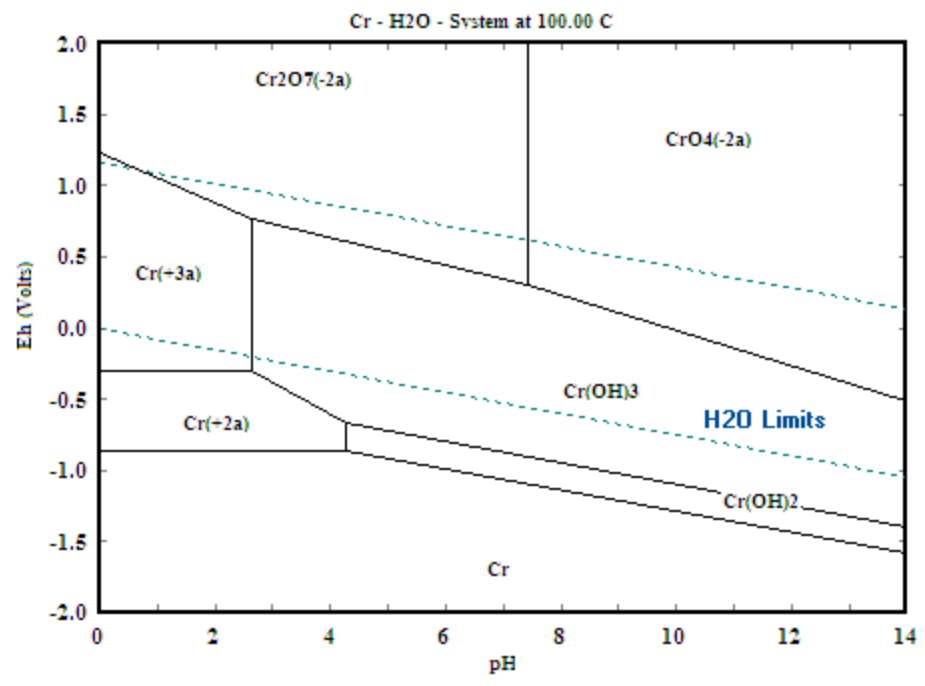

Figura 3 - Diagrama de Pourbaix para o sistema Cr-O-H.

Os dados apresentados na Figura 3 sugerem que a solubilização do cromo é termodinamicamente viável a $100^{\circ} \mathrm{C}$, dentro dos limites de estabilidade a água líquida. Tanto para valores de $\mathrm{pH}$ inferiores a 2.5 ou superiores a 11 , o cromo se apresenta, respectivamente, como $\mathrm{Cr}^{+3}$ e $\mathrm{CrO}_{4}^{-2}$. 


\section{MATERIAIS E MÉTODOS}

\subsection{Processamento químico}

Para a realização dos experimentos foi utilizado como matéria-prima finos de uma liga $\mathrm{FeCrAC}$, sendo o processo reacional definido pelo fluxograma apresentado na Figura 4 . O material foi homogeneamente misturado com quantidade controlada de $\mathrm{NaOH}$, estabelecendose um excesso de $300 \%$ em relação à condição estequiométrica. Os ensaios de ustulação foram conduzidos em fornos tubulares com atmosfera de ar comprimido $(2 \mathrm{~L} / \mathrm{min})$. O produto obtido é então lavado com água destilada a quente $\left(90^{\circ} \mathrm{C}<\mathrm{T}<100^{\circ} \mathrm{C}\right)$ visando a completa solubilização do cromato de sódio obtido. O sólido residual (material não solubilizado) é então filtrado em membrana de celulose, que é em seguida calcinada a $600^{\circ} \mathrm{C}$ durante $30 \mathrm{~min}$. O sólido final é então pesado, seguindo então para a etapa de caracterização, da mesma forma que alíquotas do licor de cromo gerado.

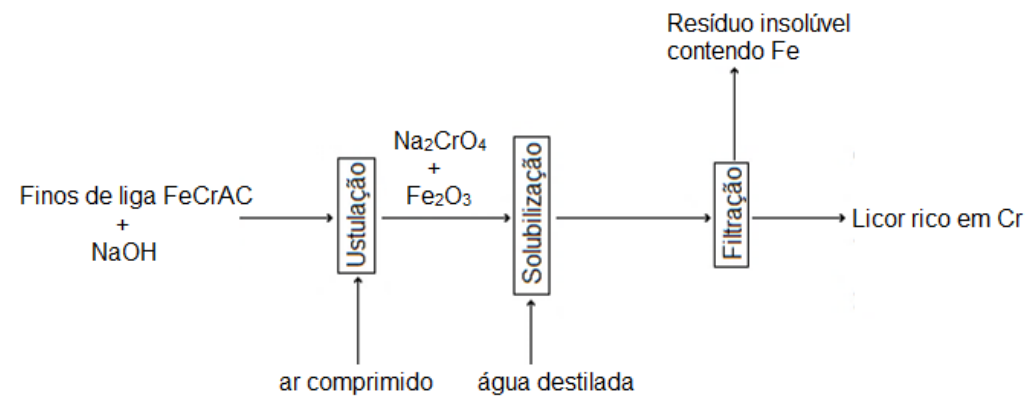

Figura 4 - Fluxograma do processo utilizado na extração do cromo.

\subsection{Métodos de caracterização}

As principais fases contidas nos finos foram determinadas via difração de raios $\mathrm{X}$ (DRX). Adicionalmente, tanto a matéria-prima inicial, quanto os produtos reacionais sólidos (material filtrado) foram caracterizados via (MEV) e espectroscopia de energia dispersiva (EDS) de maneira a se poder avaliar tanto a morfologia quanto a composição química elementar. O conteúdo de cromo total transferido para a solução foi avaliado mediante análises de espectroscopia de emissão atômica (ICP-OES). A especiação do cromo em solução foi igualmente avaliada mediante espectrofotometria de absorção na região do infravermelho (FTIR).

\section{RESULTADOS E DISCUSSÃO}

A caracterização dos finos via DRX permitiu a identificação da presença de carbetos de cromo e ferro, óxidos de ferro, aluminio, silicio, manganes, carbono (grafite), e a liga $\mathrm{Fe}-\mathrm{Cr}$ (Figura 5). Observa-se que as fases majoritárias contidas nos finos são definidas por, carbeto de cromo $\left(\mathrm{Cr}_{7} \mathrm{C}_{3}\right)-46,21 \%$, e grafite $-28,85 \%$, estando a liga $\mathrm{Fe}-\mathrm{Cr}$ associada a somente $6 \%$ da massa total da amostra. 


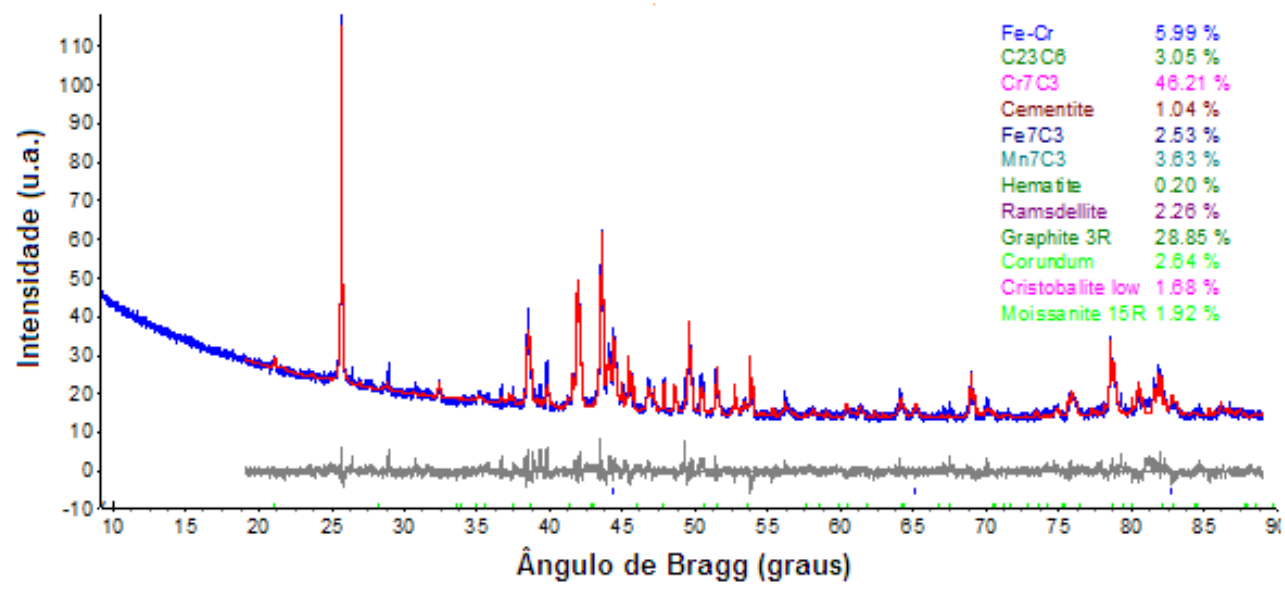

Figura 5 - Difratograma característico dos finos de liga FeCrAC.

A análise do mesmo material via MEV/EDS (Figura 6) indica a presença de partículas com morfologia homogênea, com tamanhos variando na faixa entre 10 e 150 microns. Convém notar a presença de $\mathrm{Fe}(19.07 \%), \mathrm{Cr}(44.10 \%)$ e $\mathrm{C}(22,53 \%)$, como elementos majoritários, assim como outros elementos ( $\mathrm{Mn}, \mathrm{Si}, \mathrm{Al}$ e O) presentes em com frações mássicas inferiores a $6 \%$, em concordância com a análise quantitativa do difratograma da Figura 5. Neste contexto, convém comentar que a fração mássica de $\mathrm{Cr}$ determinada via DRX (40\%) se mostra consistente com o valor determinado via EDS - 42\%, dando suporte à confiabilidade de ambas as análises.

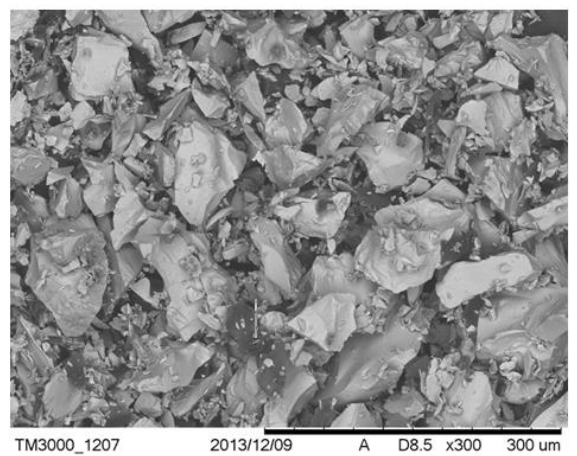

\begin{tabular}{|c|c|}
\hline $\mathrm{Cr}(\%)$ & 44,10 \\
\hline $\mathrm{Mn}(\%)$ & 5,97 \\
\hline $\mathrm{Fe}(\%)$ & 19,07 \\
\hline $\mathrm{C}(\%)$ & 22,53 \\
\hline $\mathrm{O}(\%)$ & 6,31 \\
\hline $\mathrm{Si}(\%)$ & 3,84 \\
\hline $\mathrm{Al}(\%)$ & 1,05 \\
\hline
\end{tabular}

Figura 6 - Micrografia característica e composição elementar dos finos de liga FeCrAC.

Os testes de ustulação com $\mathrm{NaOH}$ foram realizados variando-se a temperatura na faixa entre 800 e $1000^{\circ} \mathrm{C}$. Mediante a avaliação do teor de $\mathrm{Cr}$ inicialmente contido na matériaprima ("finos") via EDS, e a concentração de cromo em solução via ICP-OES, é possível calcular a recuperação de cromo global (Equação 2).

$$
R_{C r}=100\left(\frac{m_{C r}^{s o l}}{m_{C r}^{0}}\right)
$$

Onde $m_{C r}^{\text {Sol }}$ representa a massa de cromo em solução, calculada a partir do volume de água destilada utilizado $(500 \mathrm{~mL})$ e a concentração média obtida via ICP-OES, e $m_{C r}^{0}$ a massa de cromo presente nos finos. 


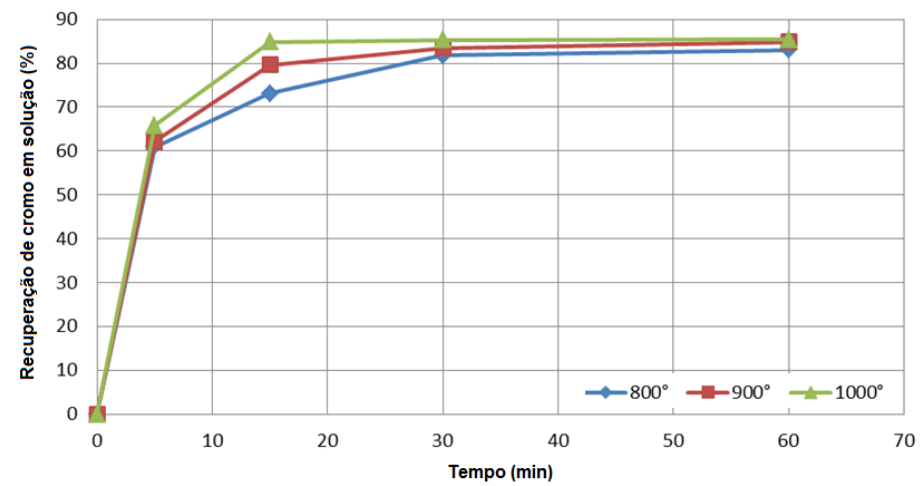

Figura 7 - Recuperação de Cr para experimentos realizados 800,900 e $1000^{\circ} \mathrm{C}$.

Os dados apresentados na Figura 7 sugerem que a etapa de ustulação de fato permitiu uma significativa conversão do cromo inicial à forma do produto reacional solúvel de interesse $-\mathrm{Na}_{2} \mathrm{CrO}_{4}$, resultando para todas as temperaturas de ustulação investigadas em recuperações superiores a $80 \%$ - tempo reacional igual ou superior a 30 minutos, sugerindo que tanto a reação de ustulação (Equação 1), quanto a solubilização em pH superior a 10 configuram processos com significativa força motriz termodinâmica, em concordância com os dados apresentados no tópico (2).

No que diz respeito às recuperações calculadas, convém observar que a elevação da temperatura na faixa investigada não apresentou influência sobre o valor final. Em contrapartida, para tempos reacionais entre 5 e 30 minutos, os dados sugerem que temperaturas mais elevadas na etapa de ustulação resultam em recuperações finais mais expressivas. De maneira a avaliar possíveis perdas de cromo durante o processo $\left(\Delta m_{C r}\right)$, foram calculadas as massas de metal presentes inicialmente, transportada para a solução e no resíduo filtrado $\left(m_{C r}^{f}\right)$ para um tempo de ustulação de 60 minutos (Tabela 1). Os dados indicam que, em todos os casos, a massa de cromo perdida (aderida à barquete e ou projetada mediante a formação e colapso de bolhas para o interior do tubo do reator) resultou em uma perda inferior a $1 \%$.

Tabela 1 - Massas de cromo presente nos diferentes materiais envolvidos

\begin{tabular}{|c|c|c|c|c|}
\hline $\mathrm{T}\left({ }^{\circ} \mathrm{C}\right)$ & $m_{C r}^{0}(\mathrm{~g})$ & $m_{C r}^{f}(\mathrm{~g})$ & $m_{C r}^{\text {sol }}(\mathrm{g})$ & $\Delta m_{C r}(\mathrm{~g})$ \\
\hline 800 & 0,164 & 0,027 & 0,136 & 0,0014 \\
\hline 900 & 0,164 & 0,023 & 0,138 & 0,0034 \\
\hline 1000 & 0,164 & 0,023 & 0,140 & 0,0012 \\
\hline
\end{tabular}


Os valores pouco expressivos de $\Delta m_{C r}$ sugerem que a limitação das recuperações calculadas (Figura 7) deve apresentar outra causa. No caso do sistema reacional em questão, a possível reação da base empregada com o material da barquete (aluminosilicato), reduziria a disponibilidade de $\mathrm{NaOH}$ para a reação de interesse (Habashi, 2002), reduzindo a quantidade de $\mathrm{Na}_{2} \mathrm{CrO}_{4}$ formado.

Finalmente, com o intuito de se estudar a especiação do cromo transportado para a solução, realizaram-se análises dos licores gerados via FTIR, sendo um resultado típico apresentado na Figura 8.

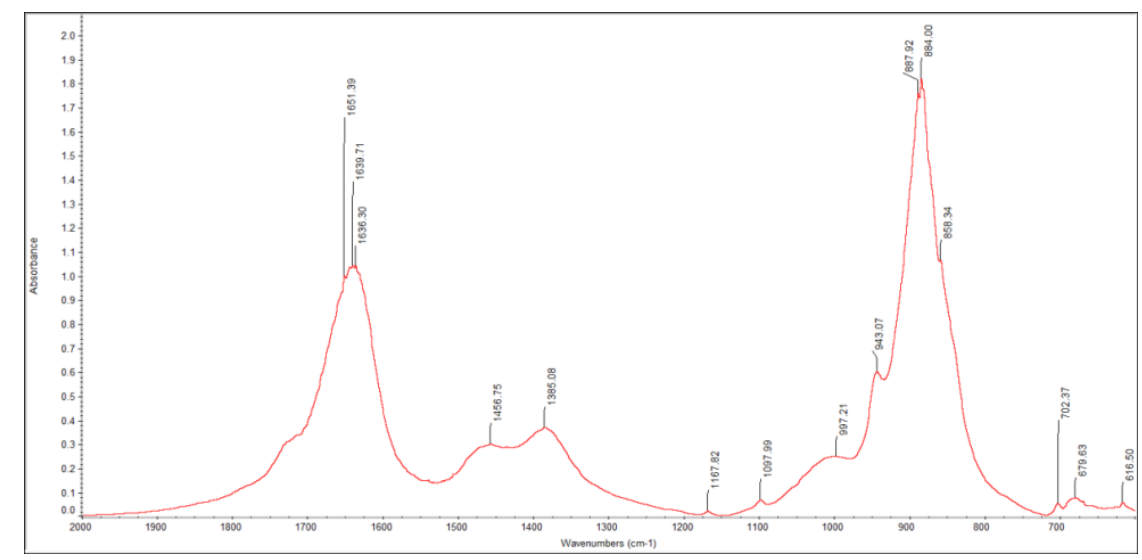

Figura 8 - FTIR do material solubilizado

Os dados contidos na Figura 8 indicam uma banda de absorção com comprimento de onda característico próximo a $940 \mathrm{~cm}^{-1}$. De acordo com Young et al. (2002), tal banda estaria associada à ligações do tipo $\mathrm{Cr}=\mathrm{O}$, servindo de evidência para a presença de ânions contendo $\mathrm{Cr}$ e O, como $\mathrm{CrO}_{4}{ }^{-2}$ e ou $\mathrm{Cr}_{2} \mathrm{O}_{7}^{-2}$, em consistência com a informação presente no diagrama de Pourbaix (Figura 3), que sugere a presença de $\mathrm{CrO}_{4}^{-2}$ para valores de $\mathrm{pH}$ superiores a 11.

\section{CONCLUSÕES}

Os dados reportados no presente trabalho indicam que recuperações de cromo superiores a $80 \%$ puderam ser alcançadas. Tal fato dá suporte a viabilidade termodinâmica de ambas as etapas envolvidas, ustulação e concomitante fusão alcalina com $\mathrm{NaOH}$ (800$\left.1000^{\circ} \mathrm{C}\right)$ e solubilização do sal $\mathrm{Na}_{2} \mathrm{CrO}_{4}$ em água destilada a quente $\left(90-100^{\circ} \mathrm{C}\right)$, conforme esperado a partir da avaliação termodinâmica apresentada no tópico (2). Os balanços de massa desenvolvidos sugerem que a limitação da quantidade de cromo efetivamente transportada para a solução deve estar associada principalmente ao consumo da base $(\mathrm{NaOH})$ mediante a reação com o material que constitui as barquetes (aluminosilicato), e não à adesão da solução nas paredes da mesma, ou via projeção da solução para o interior do tubo reacional. Adicionalmente, os dados obtidos via FTIR dão suporte à presença de espécies $\mathrm{CrO}_{4}{ }^{-2}$, que, de acordo com as expectativas termodinâmicas, deve ser o ânion de maior estabilidade para valores de $\mathrm{pH}$ superiores a 11 . Finalmente, convém comentar que os resultados satisfatórios alcançados durante a pesquisa aqui relatados serviram de motivação para a continuidade da mesma, que envolverá a redução do cromo presente em solução com $\mathrm{H}_{2} \mathrm{O}_{2}$ e subsequente precipitação do mesmo na forma de um hidróxido - $\mathrm{Cr}(\mathrm{OH})_{3}$. A calcinação do referido precipitado deve resultar na formação de $\mathrm{Cr}_{2} \mathrm{O}_{3}$, que pode ser posteriormente reduzido a cromo metálico. 


\section{AGRADECIMENTOS}

Os autores agradecem à CAPES pelo suporte financeiro e à EMPRAPA pelo suporte no que diz respeito às análises químicas via ICP-OES.

\section{RFERÊNCIAS BIBLIOGRÁFICAS}

MALAGA, W.E.L. Obtenção de óxido de cromo a partir de finos de uma liga $\mathrm{FeCr}$ alto carbono. Pontifícia Universidade Católica Rio de Janeiro, PUC Rio, 2009.

HABASHI, F. Textbook of Pyrometallurgy. Saint-Foy, Québec: Metallurgie Extractive Québec, 2002, 592p.

HABASHI, F. (Ed.) Handbook of Extractive Metallurgy. Weinheim: WILEY-VCH, 1997, 2000p.

RAMOS, M.A.F. Cromo. Sumário Mineral, DNPM. 2012.

ROINE, A. Outokumpu HSC-Chemistry for Windows, 06120-ORC-T, Versão 6.0, Outokumpu Research Oy, Information Service, Pori, Finlândia, 2006

KACHOOSANGI, R.T., COMPTON, R.G. Voltammetric Determination of Chromium(VI) using a gold film modified carbon composite electrode. Sensors and Actuators B 178, 555$562,2013$.

VANHANEM, M.R. Finnish expert report on best aviable techniques in ferrochomium production. Finnish Environment Institute, Helsinki, 1999, disponível em http://www.vmparisto.fi/default.asp?contentid. Acessado em 22/02/2014

YOUNG G. K, CHOI U.S, TAI Y. K, JUNE D. A, JIN Y.C . FT-IR and Isotherm Study on Anion Adsorption onto Novel Chelating Fibers. Macromolecular Rapid Communications V 23, 2002, p 535-539.

WENG C.H, T.Y. LIN, C.M. KAO. Enhancement of electrokinetic remediation of hyperCr(VI) contaminated clay by zero-valent iron. Journal of Hazardous Materials. v 149, 2007, p 292-302.

WENZEL, B. M. Redução carbotérmica do cromo contido nas cinzas do tratamento térmico de resíduos de couro visando a obtenção de liga ferrocromo e sulfato de cromo. Escola de engenharia, UFRGS, Porto Alegre, 2008. 